\title{
Análise dos efeitos da Terapia Instrumental Quiropráxica na postura e na força dos
}

\section{extensores do tronco}

\author{
Analysis of the effects of Instrumental Chiropractic Therapy in posture and on the strength of \\ extensors of trunk
}

Análisis de los efectos de la Terapia Instrumental Quiropráctica sobre la postura y la fuerza de los extensores del tronco

\section{Resumo}

Objetivo: Avaliar os efeitos da Terapia Instrumental Quiropráxica na postura e na forma dos músculos extensores do tronco. Metodologia: É um estudo do tipo quase-experimental de corte transversal, com uma amostra de 31 indivíduos. Foi realizada uma anamnese, em seguida a fotogrametria digital e dinamometria lombar, ambas realizadas antes e após o tratamento com a Terapia Instrumental Quiropráxica. Para análise das variáveis, foi aplicado o teste de Wilcoxon. Todas as conclusões foram baseadas em um intervalo de confiança de $95 \%$ e p <0,05. Resultados: Pode-se observar que, em relação aos dados gerais, a média de idade é de 22.10 anos e a prevalência é do gênero feminino em $71 \%$. Quanto à avaliação dinamométrica, houve um aumento de força estatisticamente significante após a intervenção $(\mathrm{p}=0.000)$. Já na avaliação postural, não houve resultados estatisticamente significantes, porém, apresentou uma resposta qualitativa sugerindo assim um alinhamento postural dos voluntários. Conclusão: A utilização da Terapia 
Instrumental Quiropráxica apresentou efeitos benéficos em relação a força da musculatura extensora do tronco, e, influenciou positivamente na auto percepção da melhora da postura corporal. Sugere-se novas pesquisas sobre o uso desta técnica para maiores evidências.

Palavras-chave: Quiropraxia; Postura; Dinamômetro de força muscular.

\begin{abstract}
Aim: Evaluate the effects of Chiropractic Instrumental Therapy on posture and shape of trunk extensor muscles. Methods: Is a study of the type of quasi-experimental cross-section, with a sample of 31 individuals. A history, then the digital photogrammetry and lumbar hand, both carried out before and after treatment with Instrumental Chiropractic Therapy. For analysis of the variables, the Wilcoxon test was applied. The conclusions were based on a $95 \%$ confidence interval and $\mathrm{p}<0.05$. Result: It can be observed that, in relation to the General data, the average age is 22.10 years and the prevalence is of female gender in $71 \%$. As for the English review, there was a statistically significant increase in strength after intervention $(\mathrm{p}=0.000)$. Already in the postural assessment, there was no statistically significant results, however presented a qualitative response suggesting a postural alignment of the volunteers. Conclusion: The use of Chiropractic Instrumental Therapy had beneficial effects in relation to the strength of the trunk extensor muscles, and positively influenced the self-perception of improvement in body posture. Further research on the use of this technique is suggested for further evidence.
\end{abstract}

Keywords: Chiropractic; Posture; Muscle strength dynamometer.

\title{
Resumen
}

Objetivo: Evaluar los efectos de la Terapia Instrumental Quiropráctica sobre la postura y la forma de los músculos extensores del tronco. Metodología: Se trata de un estudio cuasiexperimental de corte transversal con una muestra de 31 individuos. Se realizó una anamnesis seguida de fotogrametría digital y dinamometría lumbar, ambas realizadas antes y después del tratamiento con el Terapia Instrumental Quiropráctica. Para analizar las variables se aplicó la prueba de Wilcoxon. Todas las conclusiones se basaron en un intervalo de confianza del $95 \%$ y p <0,05. Resultados: Se puede observar que, en relación a los datos generales, la edad promedio es de 22,10 años y la prevalencia es femenina en $71 \%$. En cuanto a la evaluación dinamométrica, hubo un aumento estadísticamente significativo de la fuerza después de la intervención $(\mathrm{p}=0,000)$. En la evaluación postural, sin embargo, no hubo resultados estadísticamente significativos, sin embargo, presentó una respuesta cualitativa, sugiriendo así un alineamiento postural de los voluntarios. Conclusión: El uso de la Terapia Instrumental Quiropráctica tuvo efectos beneficiosos en relación con la fuerza de los músculos extensores del tronco e influyó positivamente en la autopercepción de la mejora de la postura corporal. Se sugiere realizar más investigaciones sobre el uso de esta técnica para obtener más evidencia.

Palabras clave: Quiropráctica; Postura; Dinamómetro de fuerza muscular.

\section{Introdução}

A Terapia Instrumental Quiropráxica (TIQ) baseia-se numa abordagem dinâmica que utiliza a análise de uma disfunção somática, sendo conhecida como a segunda técnica mais utilizada na quiropraxia (Clijsters, et al., 2014; Schneider, et al., 2010; Yu, et al., 2012). Estas lesões biomecânicas são isoladas e identificadas por meio dos movimentos dos pacientes, destinados a isolar e revelar as áreas exatas da disfunção. Com a detecção, o tratamento é realizado por intermédio de um instrumento mecânico projetado para fornecer um valor predefinido de força para corrigir a lesão (Cooperstein, 1997; Fuhr \& Menke, 2002). A avaliação da TIQ para a disfunção neuroarticular baseia-se no pressuposto de que o comportamento defeituoso da biomecânica articular é refletido pelas diferenças e mudanças no comprimento da perna, sendo assim o principal indicador de lesão e resposta ao tratamento (Silvestrino, et al., 2012).

Como vimos, uma das características centrais da TIQ é a análise do comprimento da perna, um procedimento comum de triagem e diagnóstico usado em quiropraxia e outras terapias manuais (Woodfield, et al., 2010). O protocolo de avaliação consiste em observações do comprimento das pernas no decúbito ventral e estímulos estressantes realizados para avaliar a função das articulações dos pés de forma progressiva e em todo o esqueleto axial (Gemmell \& Miller, 2010). O protocolo de tratamento é utilizado para determinar onde e quando, ou não, ajustar. Fica claro compreender que as diferenças dos membros inferiores podem levar a compensações que culminam com repercussões posturais. Desta maneira, o estudo da postura pode ser importante como marcador dos resultados da TIQ, sobretudo quando os pacientes já possuem disfunções posturais (Fuhr, 2009).

A postura corporal é definida como um arranjo balanceado das estruturas corporais, sendo determinada pelas posições 
dos segmentos entre si num dado momento. Em um alinhamento postural ideal, espera-se que os músculos, articulações e suas estruturas encontrem-se em estado de equilíbrio dinâmico, gerando uma quantidade mínima de esforço e sobrecarga (Żuk, et al., 2019; Schmit, et al., 2016; Cramer, et al., 2018). A avaliação postural é um método amplamente utilizado na fisioterapia para compreensão do alinhamento dos segmentos corporais, cuja finalidade é manter o corpo equilibrado, ou seja, projetar o centro de gravidade na base de sustentação, administrando assim, a força gravitacional (Poça, 2019; Santos \& Munguba, 2018; Roggia, 2016). De maneira geral, estudos relatam diversos métodos de análise biomecânica da postura em posição estática, entre estes, a fotogrametria digital (Malko, et al., 2020; Ferreira, et al., 2016). É definida como uma técnica relativamente simples, fácil e objetiva. Seu baixo custo, facilidade de foto-interpretação, alta precisão e reprodutibilidade dos resultados, além da possibilidade de arquivamento e acesso aos registros, são vantagens que justificam sua ampla utilização. Agregando informações sobre a postura, é imprescindível a análise da força. Visto que músculos fracos, hipotônicos ou espásticos podem refletir diferenças nos diversos segmentos corporais, como encontrados nas disfunções posturais (Saraiva, et al., 2020).

Sendo assim, o teste de carga dinamométrica serve para avaliarmos a força isométrica (estática) do indivíduo. Sua realização necessita de aparelhos especiais, os dinamômetros (Leonardi, et al., 2018; Nogueira, et al., 2017; Dietrich, et al., 2017).

Baseado nos fatos acima mencionados é que o presente estudo foi motivado. Os autores entendem que para favorecer maior conhecimento sobre a terapia instrumental quiropráxica, até então, com poucas referências nacionais, pode-se analisar se há real alteração na postura e também se há algum aumento de força na extensão na coluna. Desta forma, espera-se com os resultados favorecer o conhecimento da técnica, dos seus princípios e dos seus benefícios.

\section{Metodologia}

Trata-se de um estudo do tipo quase-experimental de corte transversal. Foi aprovado pelo Comitê de Ética em Pesquisa com seres humanos com número do CAAE 56390116.3.0000.5206, e $\mathrm{n}^{\circ}$ do Parecer: 1.871.884. Foi elaborado nos laboratórios especializados em Fisioterapia e Terapia Ocupacional em uma Universidade da cidade do Recife-PE. A amostra foi composta por 31 indivíduos, hígidos, de ambos os sexos. A seleção da amostra foi feita por conveniência de acordo com os voluntários que aceitaram participar do estudo. Como critérios de inclusão foram adotados: indivíduos com faixa etária entre 18-30 anos e que concordaram em participar de forma voluntária do presente estudo. Foram excluídos aqueles que apresentaram alterações motoras de origem neurológicas, alterações psiquiátricas e histórias de doenças reumáticas.

Inicialmente, os voluntários foram informados sobre os objetivos da pesquisa e sobre a sua contribuição para o estudo, após concordarem, assinaram o Termo de Consentimento Livre e Esclarecimento (TCLE). Em seguida foi realizada uma anamnese dos participantes onde constavam dados sobre nome, idade e sexo.

Posteriormente, foi realizada a avaliação, na qual foi feita uma marcação no corpo dos voluntários utilizando fita crepe ou esparadrapo em pontos anatômicos específicos: cabeça do úmero, espinha ilíaca ântero-superior (EIAS), tuberosidade anterior da tíbia (TAT) e maléolo medial. Todos esses pontos sendo marcados bilateralmente. O voluntário fícou de pé a 3 metros de distância da câmera fotográfica de 8 megapixels, onde estavam vestidos com roupas adequadas, com a distância dos maléolos mediais de $20 \mathrm{~cm}$ e com o corpo relaxado para a captura de 2 ou 3 imagens, sendo esses $20 \mathrm{~cm}$ também utilizados para calibrar a distância dos pontos no Software para Avaliação Postural (SAPO) (Alves, et al., 2017).

Os voluntários foram submetidos à avaliação postural por meio do SAPO, que é um programa de uso relativamente simples e gratuito que fornece, além das medidas lineares, valores angulares. Fundamenta-se na digitação de pontos espacialmente definidos, que possibilita funções diversas tais como a calibração da imagem, utilização de zoom, medição de distâncias e de ângulos corporais para avaliação postural, além de marcação de livres pontos, sendo este último, utilizado neste 
estudo para definição das distâncias entre a cabeça do úmero e a EIAS, EIAS e a TAT, TAT e o maléolo medial. Essas distâncias foram avaliadas ipsilateralmente e bilateralmente (Almeida, et al., 2018).

Logo após, realizaram o teste de extensão lombar utilizando o dinamômetro BACK-A da empresa TAKAI. Na dinamometria lombar o indivíduo ficou descalço, posicionado sobre a plataforma contendo uma célula de carga acoplada; permaneceu com a coluna reta mantendo os braços estendidos em frente às coxas para fazer o posicionamento correto do puxador que foi tracionado ( $1 \mathrm{~cm}$ abaixo da extremidade do dedo médio); na sequência o sujeito foi solicitado a fazer flexão anterior de tronco, com angulação de $30^{\circ}$ utilizando um goniômetro da marca CARCI, leve flexão de joelho, e segurando o puxador com as mãos será solicitado a fazer força máxima de tração no puxador, de forma perpendicular à plataforma, sem qualquer contato do puxador ou das mãos com qualquer outra parte do seu corpo (Leonardi, et al., 2018).

Em continuidade, foram realizados os Testes Básicos 1 e 2 (TB1 e TB2) para identificar o local da disfunção por meio do comprimento da perna. Os testes básicos foram realizados de acordo com os procedimentos preconizados no curso de TIQ. No TB1, com o paciente em prono, membros inferiores estendidos, emparelhados e com os pés para fora da maca, o terapeuta, com os dedos da região anterior do pé, realizava uma leve rotação da tíbia com uma eversão dos tornozelos em ambos os lados, os pés ficaram afastados em posição neutra, assim, identificamos a perna mais curta, este teste irá determinar a existência ou não de disfunção. No TB2, o paciente permanecia na mesma posição do TB1, porém, fletindo os joelhos até $90^{\circ}$ e não realizando dorsoflexão, o terapeuta irá colocar os dedos indicadores na borda lateral dos pés olhando para a linha média da planta dos pés, onde ele observava a diferença de tamanho para diagnosticar o lado e o nível da lesão. Em seguida, a intervenção foi feita pelo terapeuta que realizava de três a cinco estímulos com o aparelho na região com disfunção apontada pelo TB2 (Woodfield, et al., 2011).

Após a realização dos procedimentos, o paciente foi reavaliado com fotografia para avaliação postural e com dinamômetro para extensão lombar, para saber se houve diferenças nesses dados.

A estatística descritiva foi utilizada para determinar as medidas de tendência central e variação, tais como, número mínimo, número máximo, média, desvio padrão. Visto que os valores referentes não apresentam distribuição normal, então foi aplicado testes de hipóteses baseados em métodos não paramétricos, foi aplicado o teste de Wilcoxon, além da associação linear por linear para análises de tabela cruzada. Com isso, o teste de Wilcoxon foi utilizado para determinar se 2 populações pareadas possuíam a diferença de médias igual a 0 (zero). A associação linear por linear foi utilizada para analisar as associações entre os testes básicos presentes neste estudo. Previamente fixado o nível alpha $=0.05$ para rejeição da hipótese de nulidade. Todo o processamento estatístico foi suportado pelo software SPSS STATISTICS versão 22.0.

\section{Resultados e Discussão}

A amostra em estudo foi composta por 31 indivíduos, com idade média de $22.10 \pm 1,62$ anos, onde a grande maioria era do sexo feminino, com $71 \%$ dos casos. Na tabela 1 , foi analisada a diferença de médias na dinamometria antes e após a intervenção, onde ao avaliar a amostragem geral do estudo se obteve uma média de 70.52 e 80.13, respectivamente, de forma estatisticamente significante com o p-valor=0.000. Em relação a distribuição das médias da dinamometria entre o antes e depois dentro dos grupos feminino e masculino, nota-se que houve mudanças significativas, com p-valor de 0.000 e 0.035 , respectivamente. 
Tabela 1 - Distribuição da diferença de médias e descritiva da dinamometria antes e depois.

\begin{tabular}{|c|c|c|c|c|c|c|}
\hline Dinamometria & Variáveis & Mínimo & Máximo & Média & DP & P-Valor \\
\hline \multirow{2}{*}{ Geral } & Dinamometria Antes & 22 & 153 & 70.52 & 37.995 & \multirow{2}{*}{$0.000 *$} \\
\hline & Dinamometria Depois & 35 & 156 & 80.13 & 36.189 & \\
\hline \multirow{2}{*}{ Feminino } & Dinamometria Antes & 22 & 90 & 51.55 & 19,889 & \multirow{2}{*}{$0.000^{*}$} \\
\hline & Dinamometria Depois & 35 & 87 & 61.41 & 18,618 & \\
\hline \multirow{2}{*}{ Masculino } & Dinamometria Antes & 53 & 153 & 116.89 & 31,082 & \multirow{2}{*}{$0.035^{*}$} \\
\hline & Dinamometria Depois & 77 & 156 & 125.89 & 26,112 & \\
\hline
\end{tabular}

Nota: DP=Desvio-Padrão.

*P-Valor menor que $0.05, \log$, a diferença de média é significativa.

Fonte: Autores.

$\mathrm{Na}$ Tabela 2, foi analisada a diferença da postura entre os lados direito e esquerdo, antes e depois, de forma geral e específica, onde se obteve nenhuma associação estatisticamente significante nas distâncias analisadas pelo SAPO, porém, analisando de forma descritiva, as médias das distâncias entre os lados esquerdo e direito foram mais próximas depois da intervenção quando comparadas com as médias de antes da intervenção, sugerindo assim uma melhora no alinhamento corporal.

Tabela 2 - Diferença de médias na postura entre os lados direito e esquerdo, antes e depois.

\begin{tabular}{lcc}
\hline \multicolumn{1}{c}{ Variáveis } & Média & P-Valor \\
\hline Média Geral Direito Antes & 27.27 & 0.773 \\
Média Geral Direito Depois & 27.13 & \\
Média Geral Esquerdo Antes & 27.16 & 0.861 \\
Média Geral Esquerdo Depois & 27.03 & \\
Média Direito Antes C. Úmero-EIAS & 27.40 & 0.147 \\
Média Direito Depois C. Úmero-EIAS & 27.68 & \\
Média Direito Antes EIAS-TAT & 33.94 & 0.574 \\
Média Direito Depois EIAS-TAT & 33.67 & \\
Média Direito Antes TAT-M. Medial & 20.46 & 0.149 \\
Média Direito Depois TAT-M. Medial & 20.05 & \\
Média Esquerdo Antes C. Úmero-EIAS & 27.54 & 0.674 \\
Média Esquerdo Depois C. Úmero-EIAS & 27.67 & \\
Média Esquerdo Antes EIAS-TAT & 33.75 & 0.784 \\
Média Esquerdo Depois EIAS-TAT & 33.50 & \\
Média Esquerdo Antes TAT-M. Medial & 20.18 & \multirow{2}{*}{0.388} \\
Média Esquerdo Depois TAT-M. Medial & 19.92 & \\
\hline
\end{tabular}

Nota: C. Úmero, Cabeça do Úmero; EIAS, Espinha Ilíaca Ântero-Superior; TAT, Tuberosidade Anterior da Tíbia; M. Medial, Maléolo Medial.

Fonte: Autores. 
Na Tabela 3, ao analisar isoladamente ambos os gêneros, não houve diferença estatisticamente significante para as distâncias dos pontos anatômicos específicos, tanto antes como depois.

Tabela 3 - Diferença de médias na postura entre os grupos feminino e masculino, antes e depois.

\begin{tabular}{lcc}
\hline \multicolumn{1}{c}{ Variáveis } & Média & P-Valor \\
\hline Média Feminino Antes C. Úmero-EIAS & 27.40 & 0.465 \\
Média Feminino Depois C. Úmero-EIAS & 27.68 & \\
Média Feminino Antes EIAS-TAT & 33.94 & 0.661 \\
Média Feminino Depois EIAS-TAT & 33.67 & \\
Média Feminino Antes TAT-M. Medial & 20.46 & 0.269 \\
Média Feminino Depois TAT-M. Medial & 20.05 & 0.594 \\
Média Masculino Antes C. Úmero-EIAS & 27.54 & \\
Média Masculino Depois C. Úmero-EIAS & 27.67 & 0.236 \\
Média Masculino Antes EIAS-TAT & 33.75 & \\
Média Masculino Depois EIAS-TAT & 33.50 & 0.767 \\
Média Masculino Antes TAT-M. Medial & 20.18 & \\
Média Masculino Depois TAT-M. Medial & 19.92 & \\
\hline
\end{tabular}

Nota: C. Úmero, Cabeça do Úmero; EIAS, Espinha Ilíaca Ântero-Superior; TAT, Tuberosidade Anterior da Tíbia; M. Medial, Maléolo Medial. Fonte: Autores.

$\mathrm{Na}$ Tabela 4, ao analisar a distribuição da amostra quanto aos Testes Básicos, foi visto que no TB1 houve uma prevalência de $67.7 \%$ da Perna Curta Esquerda, já no TB2, $67.7 \%$ apresentaram como resultado do teste Curta Curta.

Tabela 4 - Distribuição da amostra quanto aos Testes Básicos.

\begin{tabular}{llcc}
\hline & Testes & Frequência & \% \\
\hline \multirow{2}{*}{ Teste Básico 1 } & Perna Curta Direita & 9 & 29.0 \\
& Perna Curta Esquerda & 21 & 67.7 \\
& Nenhuma & 1 & 3.2 \\
Teste Básico 2 & Curta Curta & 21 & 67.7 \\
& Curta Longa & 9 & 29.0 \\
& Nenhuma & 1 & 3.2 \\
\hline
\end{tabular}

Fonte: Autores.

Ao realizar a associação entre os Testes Básicos 1 e 2, nota-se uma significância com o p-valor=0.000, além disso, ambos são dependentes e que de acordo com este estudo, sugere-se que aquele que tem a Perna Curta Esquerda no TB1, provavelmente terá Curta Curta no TB2, já quem tem a Perna Curta Direita no TB1, provavelmente terá Curta Longa no TB2 (Tabela 5). 
Tabela 5 - Associação da distribuição da amostra quanto aos Testes Básicos 1 e 2.

\begin{tabular}{llcccc}
\hline & Associação & \multicolumn{3}{c}{ Teste Básico 2 } & \multirow{2}{*}{ P-Valor } \\
\cline { 3 - 5 } & Perna Curta Direita & $\begin{array}{c}\text { Curta } \\
\text { Curta }\end{array}$ & $\begin{array}{c}\text { Curta } \\
\text { Longa }\end{array}$ & Nenhuma & \\
\hline \multirow{2}{*}{ Teste Básico 1 } & Perna Curta Esquerda & 20 & 1 & 0 & \\
& Nenhuma & 0 & 0 & 0 & $\mathbf{0 . 0 0 0}^{*}$ \\
& N & 1 & 8 & 1 & \\
\hline
\end{tabular}

*P-valor menor que $0.05, \log o$, a associação é significante. Fonte: Autores.

\section{Discussão}

Ao analisar a idade, foi observado no presente estudo uma amostra de 31 indivíduos com idade média de 22.10 $\pm 1,62$ anos, parâmetro também observado no estudo dirigido por Xiaojie Yu et al. (2012) que analisava mudanças no limiar de dor e na atividade eletromiográfica basal após manipulação por instrumento na coluna, no qual obteve uma amostra de 30 indivíduos com idade média de $24.5 \pm 3,9$, porém houve uma prevalência do gênero masculino em $63 \%$, já no presente estudo a maioria foi do gênero feminino em $71 \%$. Chamamos atenção para o desvio padrão pequeno da idade (1,62 anos), o que configura um grupo homocedástico, caracterizando maior fidedignidade e harmonia nos resultados.

Quanto à mensuração da força realizada por intermédio da dinamometria, foram encontrados resultados estatisticamente significantes quando analisado a média geral antes e após a intervenção com o p-valor=0.000. Além disso, quando verificado os gêneros separadamente, também obteve-se resultados estatisticamente significantes de aumento de força tanto no masculino (p-valor=0.035) quanto no feminino (p-valor=0.000). Em um estudo realizado por Pereira et al. (2011), sobre a análise da influência da manipulação na coluna vertebral sobre a força de preensão palmar e limiar de dor, não foi observado resultados estatisticamente significantes tanto para força quanto para limiar de dor, porém os autores citam que no momento da manipulação, os ligamentos vertebrais são estirados e os proprioceptores estimulados, fazendo assim que aja um estímulo para medula espinhal, mandando como resposta um aumento no aporte sanguíneo muscular e podendo, desta forma, interferir no processo de contração muscular. Neste contexto, Humphries et al. (2013), observou que, a força da preensão palmar aumentou após a manipulação da cervical baixa. Um resultado interessante neste estudo foi que os atletas melhoraram sua performance nos arremessos do basquetebol. Parece, portanto, que realmente o controle muscular reage positivamente às manipulações, o que pode ocasionalmente servir como parâmetro para a melhora do ajuste postural.

Em relação à manipulação vertebral e postura, no estudo dirigido por Boschi e Lima (2012), sobre efeitos da manipulação torácica na dor e amplitude de movimento da coluna cervical, aborda que a manipulação vertebral irá ocasionar melhora na mobilidade articular em todos os planos, fazendo assim com que aja uma resposta no sistema esquelético, neurológico e muscular. Já em um estudo dirigido por Jorge et al. (2011), sobre o efeito da manipulação lombar em pacientes com dor lombar aguda, relata que as manipulações são capazes de equilibrar as funções musculares e viscerais. E em um estudo realizado por Silva et al. (2015), sobre os efeitos imediatos de manipulação lombar sobre a sensibilidade dolorosa e o controle postural de indivíduos com dor lombar de origem inespecífica, relata que a manipulação modifica a excitabilidade dos motoneurônios, além de desencadear reflexos musculares dos músculos paravertebrais, demonstrando um aumento na atividade eletromiográfica dos mesmos. Todos esses fatores contribuem para que a manipulação provoque um alinhamento postural.

Ademais, existem estudos como o de Gurney et al (2002), que já demonstravam uma relação importante entre a postura e o tamanho dos membros inferiores. Ele identificou que os indivíduos que apresentavam diferenças no TB1 e TB2 tinham desde assimetrias de força muscular bilateral, como alterações objetivas, como escoliose, dores articulares, processos 
degenerativos, alteração da marcha e também alterações subjetivas como parestesias e dores difusas. As alterações posturais podem decorrer também das disfunções temporomandibulares, que tem ótima resposta com a utilização do ativador mecânico (Steven et al. 2015), equivalente ao TIQ utilizado neste estudo. Provavelmente, as alterações nos captores podais, manducatórios e visuais, levam as alterações musculares, que produzem as diferenças que nós identificamos nos Testes Básicos (TB1 e TB2). O padrão deve agora ser confirmado com outros estudos, e com grupos mais numerosos de indivíduos avaliados.

$\mathrm{Na}$ avalição da postura pelo SAPO, não foi obtido resultados estatisticamente significantes quando analisado de forma geral, específica ou separada por gêneros. Pode-se sugerir que esse fato tenho ocorrido devido à diferença das distâncias avaliadas não ter sido tão grande, já que, em contrapartida, analisando qualitativamente as distâncias específicas após o tratamento com a TIQ, pode-se observar uma maior proximidade do que os números avaliados antes do tratamento, sugerindo assim, que houve algum alinhamento postural.

Nossos resultados mostram que as diferenças entre os pontos anatômicos demarcados tiveram mudanças, porém, não estatísticas. Porque será que os músculos que envolvem estas regiões reagiram dessa forma? Os autores desse estudo acreditam que as compensações musculares vieram como fator protetivo natural e como reação do corpo, como resposta aos estímulos provocados pelo TIQ. Ademais, acredita-se que a maioria das alterações posturais são decorrentes dos complexos de subluxação vertebrais, consequentes do mau posicionamento vertebral (Henderson, et al., 2007).

\section{Considerações Finais}

Com bases nos dados obtidos na presente pesquisa, pode-se demonstrar que a utilização da TIQ apresentou efeitos benéficos em relação a força da musculatura extensora do tronco, e, influenciou positivamente na auto percepção da melhora da postura corporal, além disto, mostrou-se como uma ferramenta rápida e de baixo custo. $\mathrm{O}$ estudo realizado apresentou como limitações o baixo número de voluntários, resultando em uma pequena amostragem, além da carência em artigos recentes sobre este tema. Portanto, sugere-se a realização de um ensaio clínico randomizado utilizando a Terapia Instrumental Quiropráxica para que sejam ampliadas as evidências sobre o uso desta técnica.

\section{Referências}

Almeida, I. S., Barreto, L. P., Andrade, L. S., Souza, C. V., \& Mota, Y. L. (2018). Fidedignidade nas medidas derivadas do método de palpação de um software para avaliação postural: a experiência clínica importa? Revista Brasileira de Cineantropometria \& Desempenho Humano, 20(6), 515-524

Alves, R. S., Pereira, I. C., Iunes, D. H., Rocha, C. B. J., Botelho, S., \& Carvalho, L. S. (2017). Análise intra e interavaliadores da projeção do centro de massa do corpo obtido por fotogrametria. Fisioterapia e Pesquisa, 24(4), 349-355.

Boschi, E. S., Lima, D. C. (2012). Efeitos da manipulação torácica na dor e amplitude de movimento da coluna cervical. Revista de Iniciação Científica do Unilasalle, 1(1), 78-91.

Clijsters, M., Fronzoni, F., \& Jenkins, H. (2014). Chiropractic treatment approaches for spinal musculoskeletal conditions: a cross-sectional survey. Chiropractic \& Manual Therapies, 22(1), 22-33.

Cooperstein, R. Activator methods chiropractic techinique: review. Chiropratic technique, 1997.

Cramer, H., Mehling, W. E., Saha, F. J., Dobos, G., \& Lauche, R. (2018). Postural awareness and its relation to pain: validation of an innovative instrument measuring awareness of body posture in patients with chronic pain. BMC Musculoskelet Disorders, 19(109), 1-10.

Dietrich, C.; Cardoso, J. R.; Vargas, F.; Sanchez, E. C.; Dutra, F. H.; Moreira, C.; et al (2017). Capacidade funcional em idosos e idosos mais velhos após alta da unidade de terapia intensiva. Coorte prospectiva. Revista Brasileira de Terapia Intensiva, 29(3), $293-302$.

Ferreira, A. C. A., Silva, M. R. S., Silva, E. M., \& Souza, C. E. A. (2016). Análise postural fotogramétrica em adolescentes de escola integral em Caruaru-PE. Revista Inspirar, 8(1), 50-57.

Fuhr, A. W., Menke, J. M. Activator methods chiropractic technique. Topics in Clinical Chiropractic. Academic OneFile, 2002.

Silvestrino, D., Camargo, J. V., Depintor, J. D. P., \& Lopes, S. M. L. (2012). Efetividade e eficácia do MétodoAtivadorß e do instrumento Activator - Análise crítica da literatura. Revista Brasileira de Quiropraxia, 2(118), 118-132. 
Furh, A. W. The Activator Method. (2a ed.), Elsevier; 2009.

Gemmell, H., \& Miller, P. (2010). Relative effectiveness and adverse effects of cervical manipulation, mobilisation and the activator instrument in patients with sub-acute non-specific neck pain: results from a stopped randomised trial. Chiropractic \& osteopathy, 18(1), 1-14.

Gurney, B. (2002). Leg length discrepancy. Gait and Posture, 15(1), 195-206.

Henderson, C. N., Cramer, G. D., Zhang, Q., DeVocht, J. W., \& Fournier, J. T. (2007). Introducing the external link model for studying spine fixation and misalignment: part 1 -need, rationale, and applications. Journal of Manipulative \& Physiological Therapeutics, 30(1), 239-245.

Humphries, K. M., Ward, J., Coats, J., Nobert, J., Amonette, W., \& Dyess, S. (2013). Immediate effects of lower cervical spine manipulation on handgrip strength and free-throw accuracy of asymptomatic basketball players: a pilot study. Journal of Chiropractic Medicine, 12(3), 153-159.

Jorge, S. C. F. Efeito da manipulação lombar em pacientes com dor lombar aguda [tese]. Porto: Escola Superior de Tecnologia a Saúde do Porto; 2011.67 p.

Leonardi, N. V., Kohi, L. M., Silva, L., Orsolin, E. B., Sturmer, G., Junior, N. G. B., \& Carvalho, T. G. M. L. (2018). Perfil da musculatura extensora lombar de trabalhadores rurais na atividade leiteira. Revista Brasileira de Medicina do Trabalho, 16(2), 128-135.

Malko, R. C. N., Branco, M. W., Silva, A. Z., Yamaguchi, B., \& Israel, V.L. (2020). Análise de desvios posturais em indivíduos com Doença de Parkinson avaliados pela fotogrametria. Revista Neurociências, 28, 1-14.

Nogueira, I. D. B., Nogueira, P. A. M. S., Vieira, R. H. G., Souza, R. J. S., Coutinho, A. E., \& Ferreira, G. M. H. (2017). Capacidade funcional, força muscular e qualidade de vida na insuficiência cardíaca. Revista Brasileira de Medicina do Esporte, 23(3), 184-188.

Pereira, A., Ogliari, P., Debiazi, P., Pacini, V. C., Picanço, V. V., \& Carvalho, A. R. (2011). Análise da influência da manipulação na coluna vertebral sobre a força de preensão palmar e limiar de dor. Revista Terapia Manual, 9(43), 278-283.

Poça, M. C. C. Avaliação postural em jogadoras de futebol feminino [dissertação]. Bragança: Instituto Politécnico de Bragança, Escola Superior de Saúde; 2019. 98p.

Roggia, B. (2016). Postura e equilíbrio corporal de escolares de oito a doze anos com e sem respiração oral. Revista CoDAS, 28(4), 395-402.

Santos, L. F. S., Munguba, T. A. (2018). Avaliação da postura de idosos que praticam exercícios físicos regulares. Fisioterapia Brasil, 19(5), 121-127.

Saraiva, B. M. A., Vieira, T.M., Alexandre, A. S., Araújo, G. S., Sperandio, E. F., Dourado, V. Z., Yi, L. C., \& Vidotto, M. C. (2020). Medida da Confiabilidade da Deformidade da Caixa Torácica por um Software de Avaliação Postural em Pacientes com Escoliose Idiopática do Adolescente. Revista Brasileira de Cineantropometria \& Desempenho Humano, 22, 1-12.

Schmit, E. F. D., Candotti, C. T., Rodrigues, A. P., Souza, C., Melo, M. D. O., \& Loss, J. F. (2016). Efectos del Método Pilates en la postura corporal estática de mujeres: una revisión sistemática. Fisioterapia e Pesquisa, 23(3), 329-335.

Schneider, M. J., Brach, J., Irrgang, J. J., Abbott, K. V., Wisniewski, S. R., \& Delitto, A. (2010). Mechanical vs manual manipulation for low back pain: an observational cohort study. Journal of Manipulative and Physiological Therapeutics, 33(3), 193-200.

Silva, L. S. Efeitos imediatos de uma técnica de manipulação lombar sobre a sensibilidade dolorosa e o controle postural de indivíduos com dor lombar de origem inespecífica: um ensaio clínico randomizado [tese]. Rio Grande do Sul: Universidade Federal do Rio Grande do Sul; 2015. 88 p.

Steven, D. C., Rebecca, D. C., \& Richard, D. C. (2015). Chiropractic Treatment of Temporomandibular Dysfunction: A Retrospective Case Series. Journal of Chiropractic Medicine, 14(1), 279-284.

Yu, X., Wang, X., Zhang, J., \& Wang, Y. (2012). Changes in pressure pain thresholds and basal electromyographic activity after instrument-assisted spinal manipulative therapy in asymptomatic participants: a randomized, controlled trial. Journal of Manipulative and Physiological Therapeutics, 35(6), 437-445.

Woodfield, H. C., Gerstman, B. B., Olaisen, R. H., \& Johnson, D. F. (2011) Interexaminer reliability of supine leg checks for discriminating leg-length inequality. Journal of Manipulative and Physiological Therapeutics, 34(4), 239-246.

Żuk, B., Sutkowski, M., Paśko, S., \& Grudniewski, T (2019). Posture correctness of young female soccer players. Scientific Reports, 9(1), 11179. 